\title{
An Extensible Timing Infrastructure for Adaptive Large-scale Applications
}

\author{
Dylan Stark ${ }^{1}$, Gabrielle Allen ${ }^{1}$, Tom Goodale ${ }^{1}$, Thomas Radke ${ }^{2}$, and \\ Erik Schnetter ${ }^{1}$ \\ 1 Center for Computation \& Technology, Louisiana State University, \\ 216 Johnston Hall, Baton Rouge, LA 70803, USA \\ http://www.cct.lsu.edu/, <dstark@cct.lsu.edu> \\ 2 Max Planck Institute for Gravitational Physics (Albert Einstein Institute), \\ Am Mühlenberg 1, D-14476 Golm, Germany
}

\begin{abstract}
Real-time access to accurate and reliable timing information is necessary to profile scientific applications, and crucial as simulations become increasingly complex, adaptive, and large-scale. The Cactus Framework provides flexible and extensible capabilities for timing information through a well designed infrastructure and timing API. Applications built with Cactus automatically gain access to built-in timers, such as gettimeofday and getrusage, system-specific hardware clocks, and high-level interfaces such as PAPI. We describe the Cactus timer interface, its motivation, and its implementation. We then demonstrate how this timing information can be used by an example scientific application to profile itself, and to dynamically adapt itself to a changing environment at run time.
\end{abstract}

\section{Introduction}

Profiling has long been an important part of application development. In the early days profiling was restricted to overall performance metrics such as wallclock time for a particular calculation or routine, and optimisation was often limited to finding better algorithms - ones which would take fewer operations. Today it is possible to access hardware counters which give developers information on memory metrics such as cache behaviour and floating point performance, and many tools such as SGI Speedshop, Intel's VTUNE, or the Sun Studio Performance Analyzer are available which can provide a complete performance profile of an application down to individual source lines. These tools are excellent for application developers tuning their codes, but are not useful for adaptive tuning by applications themselves, and further are limited to particular platforms or operating systems. Self-tuning of applications is becoming increasingly important in today's world of massively networked, dynamic data-driven applications [1] and the Grid computing and peta-scale applications of tomorrow.

For modern applications it is necessary to have a programming API which allows the application to query and analyze its own performance characteristics on-the-fly. It must be easy to create caliper points between which to measure 
performance and to query them, and it must be possible to access the wide range of different metrics available on modern hardware. In this paper we describe the approach taken within the Cactus framework. Cactus provides a rich timing API which can be used with basic timing metrics such as wall clock or user CPU time, or more sophisticated metrics available by plugging in libraries such as the Performance API (PAPI) [2] developed at the University of Tennessee.

The Cactus Framework [3-5] is an open source, modular, highly portable programming environment for collaborative high performance computing. Cactus has a generic parallel toolkit for scientific computing with modules providing parallel drivers, coordinates, boundary conditions, elliptic solvers, interpolators, reduction operators, and efficient I/O in different data formats. Also, generic interface definitions (e.g. an abstract elliptic solver API) make it possible to use external packages and improved modules, which are immediately available to users of the abstract interface.

Although Cactus originated in the numerical relativity community, it is now used as an enabling HPC framework for applications in many disciplines including computational fluid dynamics, coastal modeling, astrophysics, and quantum gravity. Cactus has also been a driving application for computer science research, particularly in Grid and distributed computing. For example, the socalled "Cactus-Worm" application [6] used contracts based on runtime performance to trigger migration of an astrophysics application across distributed Grid resources. Another Cactus application used MPICH-G2 to distribute a single simulation across multiple machines connected by wide area networks. Using adaptive algorithms to tune communication patterns to available bandwidth, this application showed good overall scaling [7].

By using abstract interfaces for accessing meta-information about the system state on which it is running, Cactus enables an application to be aware of its surroundings in a very portable, system-independent manner. This allows users to easily implement and experiment with dynamic scenarios, such as responding to increased delays in disk $\mathrm{I} / \mathrm{O}$ times, adapting algorithmic parameters to changes in an AMR (adaptive mesh refinement) grid hierarchy, or postponing analysis methods from in-line to a post-processing step.

In this paper, we describe the design and implementation of the Cactus timing infrastructure. Sec. 2 covers the timing infrastructure and clock API. Sec. 3 discusses how it can be used in different applications scenarios, including a new use to adaptively control checkpointing intervals for large scale simulations. In Sec. 4 the results of a case study for the checkpointing scenario are presented, and Sec. 5 compares the Cactus timing infrastructure with other packages and libraries, and explains the benefits of profiling within the application code.

\section{Cactus Timing Infrastructure}

Code using the Cactus framework is divided into modules, or components, called thorns. Each thorn declares an interface to Cactus and schedules a number of routines. Cactus controls the execution of these routines, providing a natural place 

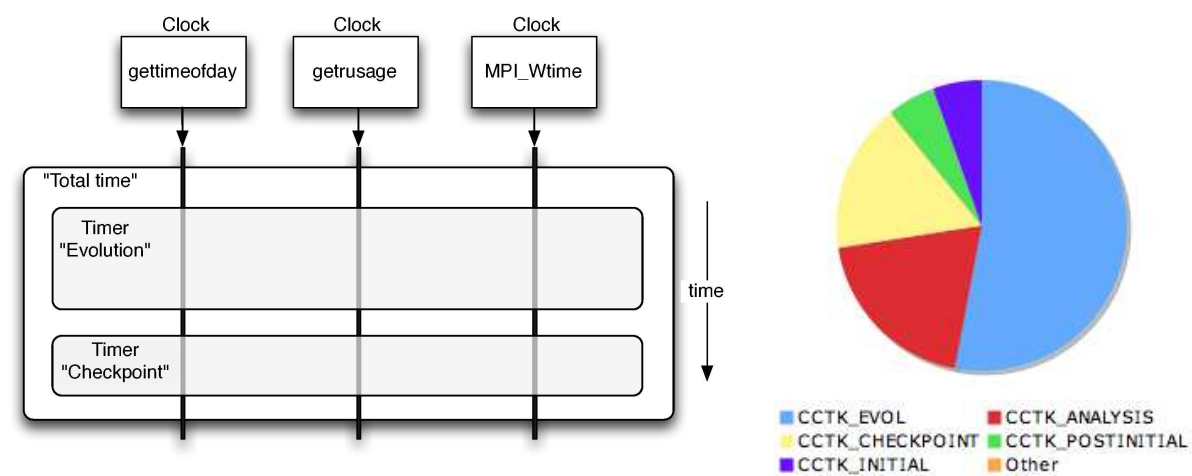

Fig. 1. Left: The relationship between timers and clocks in Cactus. Clocks are lowlevel entities representing e.g. hardware counters, timers are used by application code. Right: Example wall time distribution onto different stages of a simulation.

to put caliper points to time routines. The presence of timers in the scheduling mechanism obviates the need for developers to place explicit timers in code and allows any user or other routine to obtain timing statistics for any routine used in a particular simulation by querying the internal timer database.

Cactus provides a generic and extensible timing infrastructure. This infrastructure allows the code to access timers such as gettimeofday and getrusage, system-specific hardware clocks, and high-level interfaces such as PAPI, all in a portable manner. This timing information can be accessed programmatically at runtime through the Cactus timing API and made available to the user via online application monitoring interfaces integrated in Cactus. It is also logged semi-automatically for post-mortem review.

The Cactus timing infrastructure consists of two core concepts: timers, which are used to place caliper points around sections of code and can be switched on and off or reset, and clocks, which provide the actual timing measures, such as wall-clock time or number of floating point operations. Figure 1 shows the relationship between clocks and timers. Querying a timer returns the timing results for all clocks associated with that timer. Clocks themselves can be registered with the timing infrastructure using Cactus's standard registration techniques and thus can be provided by a thorn. This provides an extensible mechanism by which extra clocks, and hence timing metrics, can be used with no modification to any of the existing timing code.

Cactus thorns offer a wide variety of clocks. Some of these clocks are only available on certain architectures, or if certain libraries have been installed. A new clock can be easily added by providing callback functions to create, destroy, start, stop, read out, and reset the clock. Clocks are not restricted to measure time; they can measure any kind of event, e.g. discrete events such as cache misses, I/O failures, or network packet losses. Table 1 lists clocks which are 


\begin{tabular}{l|ll}
\hline Clock name & Unit Description \\
\hline gettimeofday & sec & UNIX wall time \\
getrusage & sec & UNIX system time \\
MPI_Wtime & sec & Wall time \\
PAPI counts & & Many hardware counters, e.g. instructions or Flop \\
rdtsc & & Intel CPU time stamp counter \\
\hline
\end{tabular}

Table 1. Available clocks in Cactus. Some of these clocks are only available on certain architectures, or if certain libraries have been installed.

\begin{tabular}{l|l}
\hline Function & Description \\
\hline create & Create a new clock, returning a pointer to it \\
destroy & Destroy the clock \\
start & Start this clock \\
stop & Stop this clock \\
reset & Reset this clock, i.e., set the accumulated time to zero \\
get & Get the clock's values \\
set & Set the clock's values \\
\hline
\end{tabular}

Table 2. Cactus clock API. A clock is an object which measures certain events. Several clocks of the same kind can exist and can be running at the same time, measuring potentially overlapping durations. Clock can measure several values at the same time, e.g. multiple PAPI counters. Clocks are not meant to be called by user thorns (although this is of course possible); instead, clocks are encapsulated in timers. See also Table 3.

currently available. Table 2 describes the Cactus clock API. Clocks are usually not used directly; they are instead encapsulated in timers.

The Cactus timing API is the interface which can be used to time or profile events or regions of code. Timers are usually created at startup time (or the first time a routine is entered), and they are started and stopped before and after the events that should be measured. The values of the clocks associated with a timer can be output explicitly using timer calls, or using a Cactus functionality that outputs all existing timers periodically to a log file. Table 3 gives an example of using timers, the complete API is described in the reference manual [3].

The accuracy of the timing information is obviously limited by the accuracy of the underlying clocks. Many clocks have accuracies measured in microseconds, and are hence not suitable for profiling very short events or routines. Other clocks, such as e.g. rdtsc, have nanosecond resolution and can measure with a very fine granularity. One has to keep in mind that measuring time changes the instruction flow through the CPU, often acting as barriers, so that it is impossible to measure with sub-nanosecond accuracy on today's CPU architectures.

The Cactus timer interface is a high performance interface. Creating and destroying timers typically requires allocating and freeing memory, so this should not be done in inner loops. Starting and stopping timers is as efficient as the underlying clocks implement it, plus overhead from indirect function calls. (The clocks' routines are called via function pointers.) 


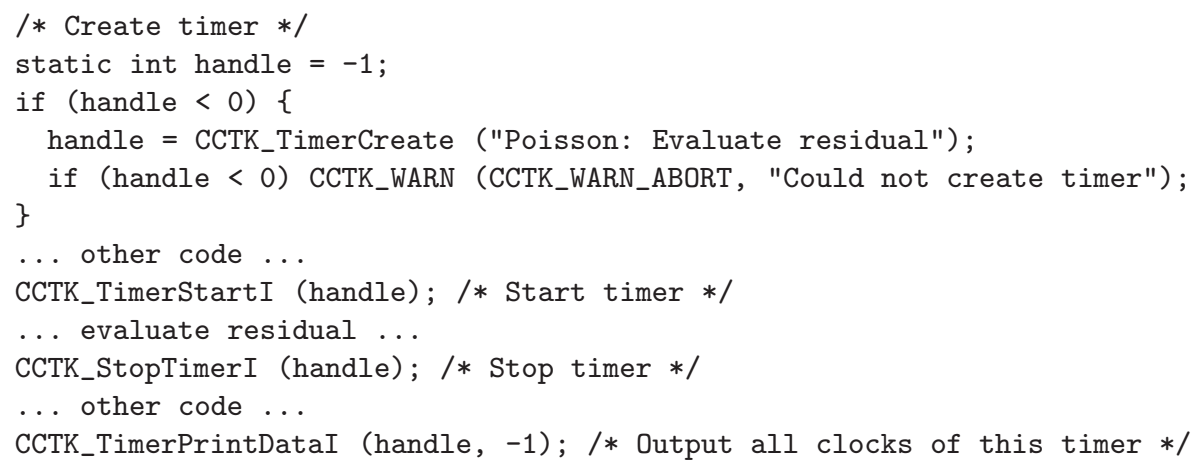

Table 3. Example source code using Cactus timers, illustrating how a timer is created, started, stopped, and printed.

\section{Use Cases}

In this section we present use cases for application self-profiling. We give two examples describing the current use of the timing infrastructure for automated report generation and the use of profiling information to guide adaptive control of applications. We also suggest some other possible application scenarios which are possible given the above timing infrastructure.

\subsection{Timer Report}

Cactus automatically sets up timers for each scheduled routine, as described in Sec. 2. The information from these timers is dynamically available to the application through the timer API. This information is used to provide details about application performance while the simulation is running, reporting it e.g. to standard output, via a web-accessible HTTP interface ${ }^{3}$, or via log files. The same mechanism can also be used to influence the behaviour of the application, allowing it to adapt itself to changes in the simulation or the environment.

Timer reports are generated for any Cactus application by setting the parameter Cactus: :print_timing_info="full". Figure 2 shows part of such a report for one of the runs of the use case presented below. The information in the report is collected by querying the timers periodically. In this case, the two clocks available to the simulation were gettimeof day and getrusage. Figure 1 shows a graphical representation of such a report.

\subsection{Adaptive Checkpointing}

Checkpointing is often used by applications deployed on clusters and supercomputers to provide protection again hardware and software failures, to allow

\footnotetext{
${ }^{3}$ See http://cactus.cct.lsu.edu:5555/TimerInfo/index.html for timing informa-
} tion for the perpetual Cactus demonstration run 


\begin{tabular}{|c|c|c|c|c|}
\hline \multirow{2}{*}{$\begin{array}{l}\text { Thorn } \\
============= \\
\text { CarpetIOHDF5 }\end{array}$} & \multirow{2}{*}{$\begin{array}{l}\text { | Scheduled routine in time bin } \\
================================ \\
\text { | Evolution checkpoint routine }\end{array}$} & \multicolumn{3}{|c|}{$\begin{array}{l}\text { | gettimeofday [secs] | getrusage [secs] } \\
=====================================0\end{array}$} \\
\hline & & I & 79.76328000 & 13.66692200 \\
\hline$============$ & 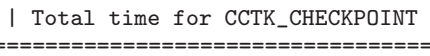 & $=$ & $\begin{aligned} & 79.76328000 \quad \mid \\
&================\end{aligned}$ & $\begin{aligned} & 13.66692200 \\
&===============\end{aligned}$ \\
\hline AdaptCheck & I Adaptive checkpointing startup & I & 0.00001300 & 0.00000000 \\
\hline BSSN_MoL & I Register provided slicings & I & 0.00000700 & 0.00000000 \\
\hline \multicolumn{5}{|c|}{ 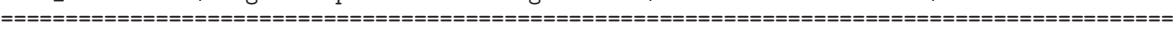 } \\
\hline & I Total time for simulation & I & 1417.13730900 । & 1305.43354400 \\
\hline
\end{tabular}

Fig. 2. Part of the standard timer report available for any Cactus application by setting a simple parameter. This report shows the time spent in some scheduled routines.

for simulations which require longer run times than available on batch queues, and more recently to enable different dynamic grid computing scenarios. Cactus provides application-level checkpointing which saves a snapshot of the running simulation by writing to file all active grid variables, parameters and other state information. The checkpoint file uses a platform independent file format, and the run can be restarted either on the same machine or on a completely different architecture using a different number of processors.

The current checkpointing mechanism in Cactus allows for checkpointing after initial data generation, periodic checkpointing based on iteration count, and checkpointing on termination. We developed a new thorn AdaptCheck which dynamically controls the checkpointing characteristics of a Cactus application using real-time profiling timing information provided through the timer infrastructure.

Writing a checkpoint file for a simulation can take a relatively long time, depending on the number of state variables to be saved, the file system characteristics, and the efficiency of I/O layer. The time needed can also vary over the lifetime of a simulation. For example, when using adaptive mesh refinement, the amount of data to be stored varies with the number of refinement levels, which itself depends on dynamic quantities such as the truncation error.

Assuming that the run is allocated some fixed amount of wall time for which it can use a resource, a growing checkpoint time would necessarily take away from the actual time spent on the problem. AdaptCheck allows the user to specify the maximum percentage of a time the simulation should spend checkpointing in order to best use the fixed amount of time available on some resource. This is a weak upper bound, which means that the thorn guarantees that no checkpoint will be performed if the current percentage of time spent checkpointing is above the specified level, but it does not guarantee that a checkpoint will not occur which will result in the percentage of time being higher than the specified level.

The quality of the fault tolerance provided by checkpointing depends on the frequency of the snapshots. Cactus currently allows the user to specify a checkpointing interval in terms of iterations, independent of the runtime performance of the simulation. Adaptive checkpointing in the manner described above could result in long periods of time without checkpointing. In order to prevent this, AdaptCheck also respects an upper bound on the length of wall time a simula- 
tion will progress without checkpointing. This guarantees that checkpoints will be generated with some regularity, with respect to wall time.

The current implementation of AdaptCheck uses the gettimeofday clock for measuring both the simulation and checkpointing durations. This will be extended to allow for the use of other user-specified clocks. We also plan to incorporate a better prediction for the time required for the next checkpoint. This will then be used to remain closer to the user-specified maximum percentage of wall time. It will also make final checkpoints reliable, which have to be finished before the queue time is used up. We present in Sec. 4 some results from tests using AdaptCheck to control checkpointing for an AMR code.

\subsection{Future Scenarios}

The previous examples illustrate two scenarios where application-side real-time profiling is used by large scale applications. Taking advantage of the flexible, well-designed timing infrastructure in Cactus, many other uses are planned.

Building on the basic timing report mechanisms described in Sec. 3.1, more advanced and informative reports can generated, for example with the web interface providing graphical interpretation of results, or automated documents could be produced in a readable format that can be easily interpreted.

The technique for adaptive checkpointing can be applied to Cactus analysis thorns, whose methods are called only when output is required. As with checkpointing, it is usual to output at regular iteration intervals, a more effective mechanism would involve choosing the output frequency dynamically based both on user requirements and performance for a particular analysis method.

In Grid computing, as new capabilities become available on production resources, taking advantage of application-oriented APIs such as the Simple API for Grid Applications [8], previously prototyped scenarios such as simulation migration, adaptive distributed simulations, task spawning, will become more regularly used. Accurate information from applications will be needed to make decisions about when and how to use such services. As described in Sec. 5, current profiling services rely on a remote service discovering and interpreting information from applications, however we believe scenarios will be more powerful and reliable when closely coupled in the application code.

For peta-scale machines, currently being deployed in the US with tens or hundreds of thousands of processors, dynamic and real-time profiling will be essential, and in particular profiling which is inherently tied into the application and automatically generated with little overhead during an application run. Current projects, in the D-Grid and US, are developing technologies for Cactus simulations to automatically produce and store profiling and application metadata from simulations. This information will then be used for analysis to lead to optimized codes and potentially improved parallel computing paradigms. 

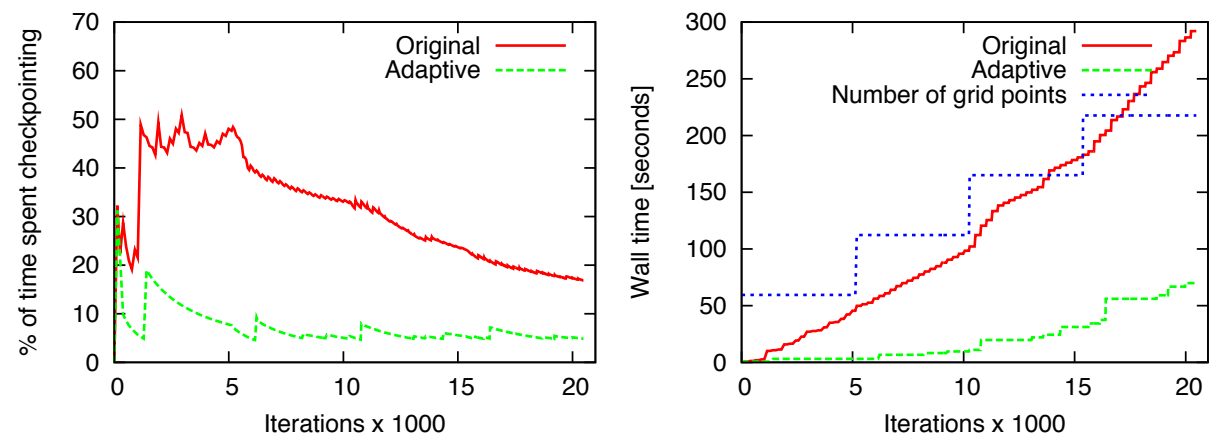

Fig. 3. Left: Percentage of time spent checkpointing during a run. The adaptive run keeps within the desired bound (5\%). Right: Total time spent checkpointing during a run. The adaptive version checkpoints less frequently to keep close to the $5 \%$ bound. The dashed line indicates the increase in the number of grid points as new refinement levels are added.

\section{Experiment and Analysis}

To illustrate the advantages of application-side adaptivity using real-time profiling a series of experiments was performed using the adaptive checkpointing described in Sec. 3.2. The application code used was the Ccatie astrophysics code [9], which can simulate the collision of black holes, to test the adaptive checkpointing functionality. This compute and data intensive application solves Einstein's equations for general relativity in 3D, evolving over twenty partial differential equations using high order finite differences. We used the Carpet driver $[10,11]$ to provide adaptive mesh refinement. Starting from a uniform grid with $40^{3}$ grid points, we added additional refined levels every 5120 iterations. Such a strategy is e.g. necessary to simulate the collapse of a stellar core in a supernova, where the central density increases considerably during the collapse. Note that for $L$ refinement levels, the computing time per iteration grows as $O\left(2^{L}\right)$, while the amount of data to be checkpointed grows at the same time as $O(L)$.

The simulation runs for 1, 737 seconds, spending $19 \%$ of the total time checkpointing. The original configuration checkpointed every 512 iterations. With the AdaptCheck thorn the maximum percentage of time spent checkpointing was restricted to $5 \%$ of the total wall time in the adaptive configuration. Figure 3 compares the actual percentage of time spent checkpointing for the original and the adaptive configuration. The results show that we are able to successfully bound this measure. This also yielded a $17 \%$ reduction in the total runtime.

Each regridding increases the problem size by $40^{3}$ grid points. This increase means that, with additional levels, each iteration takes more time to compute, the time between periodic checkpoints will increase, and the amount of time to checkpoint increases during the run. 
A common practice is to choose the checkpointing interval to be short enough at all times of the run. Unfortunately, this means that checkpointing occurs much too frequently early in the run. In another run using the AdaptCheck thorn, we bounded the interval between checkpoints independent of the performance of the run and the I/O system. This reduced the amount of time spent checkpointing from $319 \mathrm{~s}$ to $75 \mathrm{~s}$; the total runtime was reduced by $20 \%$. This functionality can be used to guarantee a certain level of fault tolerance when adapting the checkpointing based on the simulation's characteristics.

\section{$5 \quad$ Related Work and Conclusions}

The above results indicate how a scientific code can use a generic, self contained timing infrastructure for runtime profiling and adaption leading to significantly improved overall performance - in this case increasing the time spent in computation and the fault tolerance of the run, while reducing checkpointing time. The timing infrastructure was implemented in a highly portable manner in the Cactus Framework, and is easily available to users, either via parameter choices for higher level tools, or through an API for code developers. The infrastructure is able to use platform dependent clocks, as well as libraries such as PAPI.

A substantial amount of work has recently been seen in automated application profiling and adaption, motivated by new possibilities in Grid computing, and a growing realization of the new tools needed for peta-scale computing. Attention has focused on developing general libraries and tools for application profiling, adaption and steering (e.g. SciRun, GrADS, RealityGrid). For example, in the GrADS project, a program development framework has been developed which can encapsulate general applications as configurable object programs, and then optimize these for execution on a specific set of Grid resources [12]. GrADS uses the Autopilot system for real-time application monitoring and closed loop control. Autopilot sensors can be embedded in application code by developers, or as in the GrADS system an automated mechanism can be used.

The Cactus timing infrastructure incorporates its own application profiling, adaption and steering. The design of the Cactus Framework also allows thorns to be easily written to connect to external packages when these provide an advantage, as in experiments with GrADS, Autopilot, and ongoing work with SciRun. A key advantage of the Cactus infrastructure, however, is that there is an intimate connection with the scientific application - even with no attention to application profiling. Cactus applications are automatically enabled with steerable parameters and profiling at the level of thorn methods, thorns, schedule bins, as well as communication times and I/O times. Such an understanding of the application structure and scientific content is crucial for effective steering and control [13]. Higher level Cactus tools can build on these capabilities, and leverage current work on intelligent adaption in distributed environments (e.g. [14]), to provide powerful capabilities for analysis and control of scientific applications in HPC and Grid environments. 


\section{Acknowledgements}

We acknowledge contributions from the Cactus Team in the timing infrastructure implementation, in particular David Rideout, Thomas Schweizer, John Shalf, Jonathan Thornburg, Andre Werthmann, and Steve White. We thank Ed Seidel for suggestions, and Elena Caraba for her help with preparing this manuscript. This work was partly supported by NSF Grant 540179 (DynaCode) and the German Federal Ministry of Education and Research (D-Grid 01AK804). Computing resources were provided by the Center for Computation \& Technology at LSU.

\section{References}

1. Report from NSF DDDAS Workshop, January 2006, Washington. http://www. nsf.gov/cise/cns/dddas/2006_Workshop/wkshp_report.pdf.

2. The Performance API (PAPI) http://icl.cs.utk.edu/projects/papi/.

3. Cactus Computational Toolkit http://www. cactuscode.org/.

4. Goodale, T., Allen, G., Lanfermann, G., Massó, J., Radke, T., Seidel, E., Shalf, J.: The Cactus framework and toolkit: Design and applications. In: High Performance Computing for Computational Science - VECPAR 2002, 5th International Conference, Porto, Portugal, June 26-28, 2002, Berlin, Springer (2003) 197-227

5. Allen, G., Goodale, T., Lanfermann, G., Radke, T., Rideout, D., Thornburg, J.: Cactus Users' Guide. (2004)

6. Allen, G., Angulo, D., Foster, I., Lanfermann, G., Liu, C., Radke, T., Seidel, E., Shalf, J.: The Cactus Worm: Experiments with dynamic resource discovery and allocation in a grid environment. Int. J. of High Performance Computing Applications 15(4) (2001)

7. Allen, G., Dramlitsch, T., Foster, I., Karonis, N., Ripeanu, M., Seidel, E., Toonen, B.: Supporting efficient execution in heterogeneous distributed computing environments with Cactus and Globus. In: Proceedings of Supercomputing 2001, (2001)

8. Goodale, T., Jha, S., Kaiser, H., Kielmann, T., Kleijer, P., von Laszewski, G., Lee, C., Merzky, A., Rajic, H., Shalf, J.: SAGA: A Simple API for Grid Applications - High-Level Application Programming on the Grid. Computational Methods in Science and Technology 8(2) (2005)

9. Alcubierre, M., Brügmann, B., Dramlitsch, T., Font, J.A., Papadopoulos, P., Seidel, E., Stergioulas, N., Takahashi, R.: Towards a stable numerical evolution of strongly gravitating systems in general relativity: The conformal treatments. Phys. Rev. D $62(2000) 044034$

10. Schnetter, E., Hawley, S.H., Hawke, I.: Evolutions in 3D numerical relativity using fixed mesh refinement. Class. Quantum Grav. 21(6) (2004) 1465-1488

11. Adaptive mesh refinement with Carpet http://www. carpetcode.org/.

12. Berman, F., et al: New grid scheduling and rescheduling methods in the grads project. Int. J. Parallel Program. 33(2) (2005) 209-229

13. Vetter, J.S.: Experiences with computational steering on existing scientific applications. In: Parallel Processing for Scientific Computing. (1999)

14. Reed, D.A., Mendes, C.L.: Intelligent monitoring for adaptation in grid applications. Proceedings of the IEEE 93(2) (2005) 426-435 